\title{
Comparative cardiovascular risk in users versus non-users of xanthine oxidase
}

inhibitors and febuxostat versus allopurinol users

Chengsheng $\mathrm{Ju}^{*}{ }^{1}$, Rachel Wing Chuen Lai ${ }^{2}$, Ka Hou Christien Li ${ }^{2,3}$, Joshua Kai Fung Hung ${ }^{2}$, Jenny CL Lai ${ }^{4}$, Jeffery Ho PhD ${ }^{5}$, Yingzhi Liu ${ }^{6}$, Man Fung Tsoi ${ }^{7}$, Tong Liu MD PhD ${ }^{8}$, Bernard Man Yung Cheung MB BChir PhD FRCP ${ }^{7}$, Ian Chi Kei Wong $\mathrm{PhD}^{1,9}$, Lai Shan Tam MD FRCP ${ }^{2}$, Gary Tse PhD FESC FACC FRCP $\mathrm{FFPH}^{8}$

${ }^{1}$ School of Pharmacy, University College London, United Kingdom

${ }^{2}$ Laboratory of Cardiovascular Electrophysiology, Li Ka Shing Institute of Health Sciences, Hong Kong, P.R. China

${ }^{3}$ Faculty of Medicine, Newcastle University, Newcastle Upon Tyne, United Kingdom ${ }^{4}$ Department of Pharmacy \& Pharmacology, University of Bath, Bath, United Kingdom ${ }^{5}$ Department of Microbiology, Faculty of Medicine, Chinese University of Hong Kong, Hong Kong, P.R. China

${ }^{6}$ Department of Anaesthesia and Intensive Care, Faculty of Medicine, Chinese University of Hong Kong, Hong Kong, P.R. China

${ }^{7}$ Division of Clinical Pharmacology and Therapeutics, Department of Medicine, The University of Hong Kong, Pokfulam, Hong Kong, P.R. China

${ }^{8}$ Tianjin Key Laboratory of Ionic-Molecular Function of Cardiovascular disease, 
Department of Cardiology, Tianjin Institute of Cardiology, Second Hospital of Tianjin Medical University, Tianjin, 300211, P.R. China

${ }^{9}$ Centre for Safe Medication Practice and Research, Department of Pharmacology and Pharmacy, Li Ka Shing Faculty of Medicine, The University of Hong Kong, Hong Kong, Hong Kong, P.R. China

* Joint first authors

\section{Correspondence to}

Prof. Gary Tse

Professor, Tianjin Key Laboratory of Ionic-Molecular Function of Cardiovascular disease, Department of Cardiology, Tianjin Institute of Cardiology, Second Hospital of Tianjin Medical University, Tianjin, 300211, P.R. China

Email: gary.tse@ doctors.org.uk 


\section{KEY MESSAGES}

37

38

39

40

41

42

43
- Xanthine oxidase inhibitor users showed similar risks of all-cause mortality and MACE compared to non-users.

- Febuxostat users showed similar MACE and all-cause mortality outcomes compared to allopurinol users.

- Concurrent colchicine use reduced the risk of all-cause mortality and heart failure-related hospitalizations. 


\section{ABSTRACT}

Background: The aim of this study is to determine major adverse cardiovascular events (MACE) and all-cause mortality comparing between xanthine oxidase inhibitors (XOIs) and non-XOI users, and between allopurinol and febuxostat.

Methods: This is a retrospective cohort study of gout patients prescribed antihyperuricemic medications between 2013 and 2017 using a territory-wide administrative database. XOI users were matched 1:1 to XOI non-users using propensity scores. Febuxostat users were matched 1:3 to allopurinol users. Subgroup analyses were conducted based on colchicine use.

Results: Of the 13997 eligible participants, 3607 (25.8\%) were XOI users and 10390 (74.2\%) were XOI non-users. After propensity score matching, compared to non-users $(n=3607)$, XOI users $(n=3607)$ showed similar incidence of MACE (hazard ratio [HR]: 0.997, 95\% confidence interval [CI], 0.879-1.131; $\mathrm{P}>0.05)$ and all-cause mortality $(\mathrm{HR}=0.972,95 \%$ CI $0.886-1.065, \mathrm{P}=0.539)$. Febuxostat $(\mathrm{n}=276)$ users showed a similar risk of MACE compared to allopurinol users ( $n=828$; HR: 0.672, $95 \%$ $\mathrm{CI}, 0.416-1.085 ; \mathrm{P}=0.104)$ with a tendency towards a lower risk of heart failure (HF)related hospitalizations $(\mathrm{HR}=0.529,95 \%$ CI $0.272-1.029 ; \mathrm{P}=0.061)$. Concurrent colchicine use reduced the risk for all-cause mortality amongst XOI users ( $\mathrm{HR}=0.671$, $95 \%$ 0.586-0.768; $\mathrm{P}<0.001)$.

Conclusions: In gout patients, XOI users showed similar risk of MACE and all-cause mortality compared to non-users. Compared to allopurinol users, febuxostat users 
65 showed similar MACE and all-cause mortality risks but lower HF-related 66 hospitalizations.

67

68 Keywords: Xanthine oxidase inhibitor, allopurinol, febuxostat, gout, cardiovascular 69 risk, myocardial infarction 


\section{Introduction}

The burden of cardiovascular diseases (CVDs) remain high in the $21^{\text {st }}$ century particularly in low and middle income countries, where around 17 million cardiovascular deaths per year are reported [1]. Healthcare economists have estimated that, over 15 years, CVDs led to loss of 3.76 trillion US dollars, and account for than half of the economic loss due to non-communicable diseases [1]. Previous observational studies have reported a significant association between hyperuricemia or gout and increased risks of cardiovascular morbidities including congestive heart failure, myocardial infarction and cerebrovascular accident [2-5]. Therefore, understanding the cardiovascular effects of uric acid lowering drugs is important for treatment options that can reduce cardiovascular morbidity and mortality.

Xanthine oxidase inhibitors, which inhibit the conversion of purines to uric acid, are commonly prescribed for gout patients $[6,7]$. They are indicated in the treatment of gout to achieve urate target levels and to cure signs and symptoms of inflammatory response to deposition of urate crystals within the joints [8]. Their use is associated with reduced production of superoxide species and intracellular oxidative stress, leading to potentially improved endothelial and cardiac function $[9,10]$. Recognition of this molecular mechanism prompted researchers to conduct observational studies [11-15] and clinical trials [16] to examine potential cardioprotective effects of xanthine oxidase inhibitors.

However, studies reporting on the association between exposure to gout 
medications and cardiovascular risk have demonstrated conflicting results. In a Danish population study involving more than 65,000 hyperuricemic patients revealed $11 \%$ reduced risk for adverse cardiovascular events in allopurinol users compared to propensity score matched non-users [14]. No association was observed in a general gout patient cohort from the United States insurance claim database [15]. A randomized controlled trial conducted in the United States found that allopurinol did not improve clinical status, exercise capacity, quality of life, or left ventricular ejection fraction after 24 weeks of treatment in hyperuricemic patients with heart failure [17]. Another study even reported an elevated cardiovascular risk with allopurinol users using a cohort from a Taiwan-based insurance database [18].

Moreover, to date, the majority of studies have been limited to allopurinol $[6,14$, 19] until the launch of the Cardiovascular Safety of Febuxostat and Allopurinol in Patients With Gout and Cardiovascular Morbidities (CARES) trial [16]. The finding of higher all-cause mortality including cardiovascular mortality in patients prescribed with febuxostat led to an issue of public safety alert by the U.S. Food and Drug Administration (FDA). However, the results were limited by the substantial loss-offollow-up rate $(45.0 \%$ overall, $45.0 \%$ in the febuxostat group and $44.9 \%$ in the allopurinol group). In Europe, The European Union Risk Management Plan for febuxostat has called for a pharmacovigilance plan with a large cardiovascular safety investigation of febuxostat, Febuxostat versus Allopurinol Streamlined trial (FAST). This ongoing investigation is in response to reports of cardiovascular events during its Phase III randomised controlled trials and long-term clinical extension studies, where 
$60 \%$ of the cohort had $\geq$ two cardiovascular risk factors [20]. Both trials address the comparative safety between allopurinol and febuxostat, whether the use of XOI agent has benefits compared to placebo remain unanswered [21]. Therefore, current studies assessing the effect of anti-hyperuricemia treatments, including XOI, on cardiovascular diseases are inconclusive until the results from the ongoing RCTs prove otherwise [22, 23]. cardiovascular events and all-cause mortality between XOI users and non-users, and cardiovascular events in a real-world setting in Hong Kong.

\section{Methods}

\section{Study design and data sources} representative sample derived from the Clinical Data Analysis and Reporting System, an electronic health record system managed by the Hong Kong Hospital Authority. This is a computerized regional electronic health database linking all the patients' health records under primary, secondary, and tertiary care provided by 121 governmentsubsidized out-patient clinics, and 43 acute and sub-acute hospitals. These serve more than $90 \%$ of the local population and were used for producing high quality research by pharmacoepidemiologic studies from local research teams [24-28]. 
Clinical data from the database include patient-specific personal information,

diagnosis, procedure, prescription, laboratory test results, admission and discharge information. Patient information are coded using reference numbers and hospital numbers to protect patient confidentiality. Ethics approval was obtained from The Joint Chinese University of Hong Kong-New Territories East Cluster Clinical Research Ethics Committee.

\section{Study population and cohort definition}

Over a search period of five years $\left(1^{\text {st }}\right.$ January 2013 to $31^{\text {st }}$ December 2017$)$, we recruited in-patients who had a diagnosis of gout or were prescribed xanthine oxidase inhibitors admitted to nine hospitals and out-patients from 47 clinics offering community-based primary care healthcare services. A case of gout was defined as having principal diagnosis with any of the following International Classification of Diseases, Ninth Revision, Clinical Modification (ICD-9 CM) codes: 274.0 (gouty arthropathy), 274.10 (gouty nephropathy, unspecified), 274.81 (gouty tophi of ear), 274.89 (gout with other specified manifestations) and 274.9 (gout, unspecified).

cohort. Patients with no exposure to XOI were defined as the comparator cohort. Among the XOI user cohort, febuxostat users (febuxostat $40 \mathrm{mg} / 80 \mathrm{mg} / 120 \mathrm{mg}$ daily) and allopurinol users (allopurinol $100 \mathrm{mg} / 200 \mathrm{mg} / 300 \mathrm{mg}$ daily) were identified on the intention-to-treat basis and were defined as the febuxostat user cohort and the 
allopurinol cohort, respectively. Patients under 18 years old or had a history of MACE or heart failure before the first gout diagnosis were excluded from the study cohorts. Patients initiated any XOI before the first gout diagnosis record were further excluded.

\section{Outcomes and follow-up}

The primary analytic outcome was taken to be a composite of hospitalisation due to heart failure and the 3-point major adverse cardiovascular event (MACE) defined by FDA, including nonfatal infarction, nonfatal stroke and cardiovascular death. The secondary outcome was all-cause mortality. Analyses for both outcomes were made by comparing XOI user cohort versus XOI non-user cohort as primary analyses, and febuxostat user cohort versus allopurinol user cohort as secondary analyses. The primary cause of death was recorded as ICD-10 CM code in the Clinical Data Analysis and Reporting System. The index date for the XOI non-user cohort was defined as the date of diagnosis of gout. The index date for the XOI user cohort, including the allopurinol and febuxostat user subgroups, was defined as the initiation of XOIs, whether allopurinol or febuxostat. In order to address the issue of potential immortal time bias, the patient-years of XOI users between the diagnosis of gout and the start of XOI treatment were included in the XOI non-user cohort for any analyses. All patients were followed from the index data either the occurrence of the primary outcome, death or the last day of search, whichever occurs first. 


\section{Covariates}

In order to control potential confounders, the following baseline characteristics were obtained at the index admission: age, sex, comorbidities (peripheral vascular diseases, rheumatic diseases, liver diseases, diabetes mellitus and renal diseases), Deyo's Charlson Comorbidity Index, concurrent long-term cardiovascular medications (beta blockers, diuretics, lipid-lowering drugs, anticoagulants, anti-platelets, calcium channel blockers, antidiabetic drugs, nitrates, angiotensin converting enzyme inhibitors and angiotensin II receptor blockers) and other gout medications (non-steroid antiinflammatory drugs, probenecid and colchicine). Deyo's Charlson comorbidity score was calculated based on ICD-9 CM using 17 major medical conditions, such as dementia, malignancy, diabetes, renal and hepatic diseases.

\section{Subgroup analyses}

We conducted two subgroup analyses based on colchicine use. The first subgroup analyses were stratified by colchicine use. In the second subgroup analyses, we defined the stratification as whether individual patients have received a colchicine prescription with the duration longer than 3 days. All subgroups were split from the main matched cohort without further matching $[29,30]$. Hazard ratios for both primary and secondary outcomes were calculated for two subgroup analyses. Following the subgroup analyses, we conducted further analyses to investigate the effect of concurrent colchicine on the primary and secondary outcomes within the XOI user cohort. 
Statistical analysis

To minimize bias by confounding, propensity score matching was applied to adjust all baseline covariates. The propensity scores were generated by multivariate logistic regression using all variables listed in Table 1. In primary analyses, XOI users were matched with XOI non-users at a fixed 1:1 ratio. In secondary analyses, febuxostat users were matched with allopurinol users a fixed 1:3 ratio. Both matchings were performed by the nearest-neighbour matching method. Baseline characteristics between the matched cohorts were compared using both P-value and standardized mean difference.

Pearson's Chi-square test or Fisher's exact test were used to compare between groups for categorical variables as appropriate. Considering normality, Student's t-test or Mann-Whitney $U$ test were applied for continuous variables. Time-to-event analysis was conducted using Cox proportional hazards regression. Crude hazard ratios (HR) were presented in $95 \%$ confidence intervals for each outcome. Time-dependent covariates were evaluated to ensure our models did not violate the assumption of proportional hazards. Propensity score matching was conducted with R statistical software version 3.5.2 and all other statistical analyses were conducted using Statistical Package for Social Sciences. (IBM SPSS version 25.0, Armonk, NY). Two-sided Pvalues of less than 0.05 were considered statistically significant.

\section{Results}


Study population

Of the 20114 patients identified, 15696 (78.0\%) subjects did not have a history of MACE or heart failure before a diagnosis of gout was made. Based on drug dispensing records, 5306 XOI users were identified. To identify the patients who are XOI-naïve, those receiving XOI treatment before the first gout diagnosis were excluded $(n=1699)$. Thus, the remaining XOI users $(n=3607)$ were propensity score (PS)-matched in a 1:1 ratio to XOI non-users. Moreover, 276 intention-to-treat febuxostat users and 3331 intention-to-treat allopurinol users were identified from 3607 eligible XOI users. Febuxostat users were then matched 1:3 with allopurinol users. The selection of study population is summarized in Figure 1.

The baseline characteristics of the unmatched cohorts are detailed in Supplementary Table 1. Compared to XOI non-users, XOI users tended to have more male sex, younger age, more pretreatment renal conditions, diabetes mellitus, higher Charlson's Comorbidity Index Score and more concurrent uses anti-diabetic medications, angiotensin converting enzyme inhibitors/angiotensin II receptor blockers, beta-blockers, calcium channel blockers, anticoagulants/antiplatelet agents and less NSAIDs. Furthermore, among XOI users, febuxostat users tended to have higher Charlson's Comorbidity Index Score, more concurrent uses of antidiabetic medications, diuretics, colchicine and probenecid than allopurinol users.

The baseline characteristics of the study population after 1:1 and 1:3 PS-matching are shown in Table 1. All the baseline covariates were well-matched between the XOI 
users and non-users. For the matched febuxostat and allopurinol users, probenecid use was relatively imbalanced $(\mathrm{SMD}=0.197)$ after matching due to that limited records were found from the allopurinol users. The median (IQR) follow-up time was 1.97 (0.75, 3.57) years for the XOI users and $1.85(0.71,3.36)$ years for the XOI non-users after matching. For the secondary analysis cohorts, it was $1.50(0.60,2.58)$ years for the febuxostat users and $1.96(0.79,3.48)$ years for the allopurinol users after matching.

\section{Primary analyses}

Comparing to XOI non-users, XOI users had similar a risk of meeting the primary outcome (Hazard Ratio $=0.997,95 \%$ CI 0.879-1.131; $\mathrm{P}=0.962$ ) (Figure 2A). Separate analyses were conducted for the individual component of the primary outcome (Table 2). No significant difference was observed between XOI users and XOI non-users for non-fatal MI $(\mathrm{HR}=1.111,95 \%$ CI $0.849-1.453 ; \mathrm{P}=0.444)$, congestive heart failure $(\mathrm{HR}=1.028,95 \%$ CI $0.870-1.215 ; \mathrm{P}=0.743)$, cardiovascular death $(\mathrm{HR}=0.680,95 \% \mathrm{CI}$ $0.216-2.145 ; \mathrm{P}=0.511)$ or stroke events $(\mathrm{HR}=0.821,95 \%$ CI $0.640-1.054 ; \mathrm{P}=0.121)$. For the secondary outcome, XOI users did not have a significant difference in all-cause mortality when compared to non-users $(\mathrm{HR}=0.972,95 \% \mathrm{CI} 0.886-1.065 ; \mathrm{P}=0.539)$

(Figure 2B).

\section{Secondary analyses}

Allopurinol users showed similar rates of the primary outcome, individual MACE 
outcomes, and all-cause mortality when compared to XOI non-users (Supplementary

Table 2). Febuxostat use was associated with a similar risk of MACE and heart failure $(\mathrm{HR}=0.672,95 \%$ CI $0.416-1.085 ; \mathrm{P}=0.104)$ (Figure 3A). Analyses for individual components of the primary outcome revealed no significant association but the risk for congestive heart failure tended to be lower $(\mathrm{HR}=0.529,95 \%$ CI $0.272-1.029 ; \mathrm{P}=0.061)$ (Table 3). Febuxostat users showed a similar all-cause mortality compared to allopurinol users $(\mathrm{HR}=0.985,95 \%$ CI 0.725-1.338; $\mathrm{P}=0.921)$ (Figure 3B).

\section{Subgroup analyses}

The first subgroup analysis on colchicine use was conducted. Among 5277 colchicine users, there were 2628 XOI users and 2649 XOI non-users. No significant difference in the primary or secondary outcome was observed. Among the 1937 colchicine non-users, 979 patients were from the matched XOI user cohort and 958 were from the matched XOI non-user cohort. Whilst there was no difference in the risk for primary outcome between either group, XOI users showed a higher incidence of heart failure-related hospitalizations $(\mathrm{HR}=1.434,95 \% \mathrm{CI} 1.015-2.026 ; \mathrm{P}=0.041)$ but a lower risk in all-cause mortality $(\mathrm{HR}=0.849,95 \%$ CI $0.728-0.990 ; \quad \mathrm{P}=0.036)$

\section{(Supplementary Table 3).}

The second subgroup analysis was conducted for duration of colchicine prescription. Of these, 4082 patients with prescriptions $>3$ days and 3132 patients with prescriptions less than this duration. Among the 4082 patients with prescriptions $>3$ days, 2201 were 
XOI users and 1881 were XOI non-users and no difference were found in risk of primary or secondary outcome. Among those 3132 patients with prescriptions $<=3$ days , 1406 were XOI users and 1726 were XOI non-users. The XOI users had a higher risk for heart failure-related hospitalizations ( $\mathrm{HR}=1.353,95 \% \mathrm{CI} 1.045-1.750 ; \mathrm{P}=0.022)$, but no difference in the primary or secondary outcome, or individual components of the primary outcome (Supplementary Table 4).

Analysis on the effect of concurrent use of colchicine within the matched XOI cohort $(n=3706)$ was conducted. We compared 2628 colchicine users with 979 colchicine non-users. The colchicine users had a significantly lower risk for all-cause mortality $(\mathrm{HR}=0.671,95 \% 0.586-0.768 ; \mathrm{P}<0.001)$ and a similar risk for the primary outcome including each individual cardiovascular endpoint. 2201 XOI users with prescription of colchicine $>3$ days were compared to 1406 XOI users with prescriptions $<=3$ days. Consistently, colchicine users with longer duration ( $>3$ days vs. $<=3$ days) showed a lower risk for all-cause mortality $(\mathrm{HR}=0.600,95 \% \mathrm{CI} 0.527-0.683 ; \mathrm{P}<0.001)$. The risk for the primary outcome was lower amongst these colchicine users $(\mathrm{HR}=0.813$, 95\% CI $0.680-0.972 ; \mathrm{P}=0.023$ ), which was mainly driven by a lower risk for heart

\section{Discussion}


The main findings of this retrospective study of gout patients using electronic health records are that i) no benefit of XOI use on risk of major cardiovascular events or all-cause mortality; ii) febuxostat treatment was associated with a similar risk of cardiovascular events and all-cause mortality compared to allopurinol treatment; iii) concurrent colchicine use reduced the risk for all-cause mortality amongst XOI users; and iv) colchicine use longer than 3 days significantly reduce heart failure-related hospitalizations.

The null association between XOI treatment and cardiovascular events and allcause mortality has been reported in previous cohort studies, and confirmed by systematic reviews and meta-analyses of randomized controlled trials [4, 31, 32]. A cohort study conducted using the U.S. insurance claims-based data reported that XOI users did not have a different cardiovascular risk compared to patients with untreated hyperuricemia [33], a finding that is supported by our results. The crude incidence rates of individual cardiovascular endpoints in this study were also similar to the results reported in other studies [13]. A meta-analysis of 91 randomized controlled trials comparing the use of XOI with placebo has also shown similar results for MACE $(\mathrm{OR}=0.71,95 \% \mathrm{CI} 0.46-1.09)$ and death $(\mathrm{OR}=0.89,95 \%$ CI $0.59-1.33)$ comparing to our study [4]. Moreover, The EXACT-HF study failed to demonstrate benefits of allopurinol in patients with symptomatic HF [17]. Therefore, this study adds value to the existing literature that xanthine oxidase inhibition does not produce significant cardiovascular benefits [34-36]. 

different risk of MACE or all-cause mortality compared to allopurinol users. These findings are in keeping with those of previous studies. In the large scale, randomized, double-blinded, non-inferiority CARES clinical trial [37], febuxostat demonstrated noninferiority to allopurinol with respect to rates of adverse cardiovascular events while all-cause mortality and cardiovascular mortality were higher with febuxostat. There are potential reasons as to why this was the case. There was a significant drop-out rate (45\%) of both groups and premature discontinuation could bias towards the null for safety outcomes [38]. Furthermore, post hoc ascertainment led to the addition of more deaths to the allopurinol than to the febuxostat group, which would render the original hazard ratio non-significant $(\mathrm{HR}=1.09,95 \%$ CI 0.94-1.28)[38]. Lastly, the CARES trial was restricted to use of XOIs in secondary prevention while we examined outcomes in primary prevention. Due to these reasons, the results from CARES trial have generated heated debates regarding their application to the wider patient populations with gout [21]. Thus, in this regard, population-based cohort studies have offered a complementary approach to answer clinical questions. Thus, a large scale populationbased cohort study conducted using US Medicare claims data comparing febuxostat and allopurinol uses in gout patients has shown the no difference in composite cardiovascular events, heart failure and all-cause mortality [13].

Notably, our febuxostat user cohort has numerically lower incidence for cardiovascular outcomes $(\mathrm{HR}=0.67)$ and congestive heart failure $(\mathrm{HR}=0.53)$ than allopurinol users. Similar results were reported in previously conducted studies. In one 
observational study investigating XOI use in gout patients with stage 3 or 4 chronic

347 kidney disease, those taking febuxostat were less likely to experience major

348 cardiovascular events defined as coronary artery disease, cerebrovascular disease, and

349 peripheral vascular disease and heart failure [5]. In a Korean nationwide cohort study,

350 the composite cardiovascular endpoint of hospitalisation for myocardial infarction,

351 stroke/transient ischaemic attack, or coronary revascularisation tended to be higher with

352 allopurinol than with febuxostat [39]. An recently published observational study,

353 febuxostat demonstrated a better cardioprotective effect than allopurinol in patients

354 with heart failure [40]. Another randomized, double-blind, parallel between-group,

355 comparative trial conducted in China also showed that the urate-lowering efficacy of

356 daily febuxostat $80 \mathrm{mg}$ was greater than that of febuxostat $40 \mathrm{mg}$ and allopurinol 300

$357 \mathrm{mg}$, which exhibited comparable urate lowering efficacy [37]. The underlying

358 mechanistic explanation to the difference in cardio-protective effects between

359 febuxostat and allopurinol has been reported in various studies. Febuxostat selectively

360 inhibits both oxidized and reduced forms of xanthine oxidase and has minimal effects

361 on other enzymes of purine and pyrimidine metabolism [7]. It has been demonstrated

362 that febuxostat has superior antioxidant and anti-inflammatory effects than allopurinol

363 [41, 42]. Clinically, febuxostat has demonstrated a stronger urate-lowering effect than

364 allopurinol [43]. Since serum uric acid is an independent risk factor for cardiovascular

365 diseases [3], a greater degree of uric acid lowering by febuxostat leads to lower

366 cardiovascular risks in patients than allopurinol. 
colchicine with XOI could reduce the risk for all-cause mortality. Long-term use of colchicine could ameliorate the effect of XOI on new-onset heart failure. This could be due to the possible anti-inflammatory effect of colchicine. Our study found that colchicine use reduced heart failure-related hospitalizations. In one prospective randomised study investigating the effect of colchicine in stable chronic heart failure, colchicine effectively reduced the level of inflammatory biomarkers but without improvement in cardiac function of patients [44]. A meta-analysis found that colchicine alone did not exert any benefit on the prevention of heart failure [45]. One cohort study found that colchicine reduced the risks for cardiovascular events and all-cause mortality in gout patient [46]. While there is much uncertainty on the cardiovascular effect of with concurrent XOI usage.

\section{Strengths and limitations of this study}

This study was strengthened by extracting all eligible cases over the study period based on pre-defined billing codes followed by propensity score matching to generate a population representative sample to minimize confounding bias. In order to minimize the bias imposed by the presence of otherwise high-risk individuals, patients with the history of MACE before the index date of diagnosis with gout were excluded hence all 387 study subjects were in primary prevention. Third, our study population was homogenous Chinese, our study was free from confounding by ethnicity. 

alcohol drinking histories, body mass index, use of over-the-counter medicines and adherence to prescribed medications, which are potential confounders. Second, insufficient laboratory results were recorded, especially the serum urate level after drug treatments, which restricted us from conducting a more comprehensive analysis. Third, Severity of gout could not be assessed from the database, which is particularly concerning as we compared XOI users to non-users. Last, the small number of febuxostat users in the secondary analyses rendered insufficient statistical power.

\section{Conclusion} compared to non-users. Compared to allopurinol users, febuxostat users showed similar MACE and all-cause mortality risks but lower HF-related hospitalizations.

\section{Conflicts of interest}

405

There are no competing interests to declare.

\section{Disclosure}

409 No disclosures.

\section{Funding}

412 None. 
4151 Laslett LJ, Alagona P, Jr., Clark BA, 3rd, et al. The worldwide environment of cardiovascular 416 disease: prevalence, diagnosis, therapy, and policy issues: a report from the American College of 417 Cardiology. Journal of the American College of Cardiology 2012;60(25 Suppl):S1-49.

4182 Strasak AM, Kelleher CC, Brant LJ, et al. Serum uric acid is an independent predictor for all 419 major forms of cardiovascular death in 28,613 elderly women: a prospective 21 -year follow-up 420 study. Int J Cardiol 2008;125(2):232-9.

4213 Kawai $T$, Ohishi M, Takeya $Y$, et al. Serum uric acid is an independent risk factor for 422 cardiovascular disease and mortality in hypertensive patients. Hypertens Res 2012;35(11):1087 -92.

4234 Bredemeier M, Lopes LM, Eisenreich MA, et al. Xanthine oxidase inhibitors for prevention of 424 cardiovascular events: a systematic review and meta-analysis of randomized controlled trials. BMC 425 Cardiovasc Disord 2018;18(1):24.

4265 Foody J, Turpin RS, Tidwell BA, Lawrence D, Schulman KL. Major Cardiovascular Events in 427 Patients with Gout and Associated Cardiovascular Disease or Heart Failure and Chronic Kidney 428 Disease Initiating a Xanthine Oxidase Inhibitor. Am Health Drug Benefits 2017;10(8):393 -401.

4296 Kelkar A, Kuo A, Frishman WH. Allopurinol as a cardiovascular drug. Cardiol Rev 430 2011;19(6):265-71.

4317 Schumacher HR, Jr., Becker MA, Wortmann RL, et al. Effects of febuxostat versus allopurinol 432 and placebo in reducing serum urate in subjects with hyperuricemia and gout: a 28 -week, phase 433 III, randomized, double-blind, parallel-group trial. Arthritis Rheum 2008;59(11):1540-8.

4348 Richette P, Doherty M, Pascual E, et al. 2016 updated EULAR evidence-based 435 recommendations for the management of gout. Annals of the Rheumatic Diseases 2017;76(1):29 43642.

4379 Melendez-Ramirez G, Perez-Mendez O, Lopez-Osorio C, Kuri-Alfaro J, Espinola-Zavaleta N. 438 Effect of the treatment with allopurinol on the endothelial function in patients with hyperuricemia. 439 Endocr Res 2012;37(1):1-6.

44010 Tse G, Yan BP, Chan YW, Tian XY, Huang Y. Reactive Oxygen Species, Endoplasmic Reticulum 441 Stress and Mitochondrial Dysfunction: The Link with Cardiac Arrhythmogenesis. Front Physiol $442 \quad 2016 ; 7: 313$.

44311 Struthers AD, Donnan PT, Lindsay P, McNaughton D, Broomhall J, MacDonald TM. Effect of 444 allopurinol on mortality and hospitalisations in chronic heart failure: a retrospective cohort study. 445 Heart 2002;87(3):229-34.

44612 Wei L, Mackenzie IS, Chen Y, Struthers AD, MacDonald TM. Impact of allopurinol use on urate 447 concentration and cardiovascular outcome. Br J Clin Pharmacol 2011;71(4):600-7.

44813 Zhang M, Solomon DH, Desai RJ, et al. Assessment of Cardiovascular Risk in Older Patients 449 With Gout Initiating Febuxostat Versus Allopurinol. Circulation 2018;138(11):1116-26.

45014 Larsen KS, Pottegard A, Lindegaard HM, Hallas J. Effect of Allopurinol on Cardiovascular 451 Outcomes in Hyperuricemic Patients: A Cohort Study. Am J Med 2016;129(3):299-306 e2.

45215 Kim SC, Schneeweiss S, Choudhry N, Liu J, Glynn RJ, Solomon DH. Effects of xanthine oxidase 453 inhibitors on cardiovascular disease in patients with gout: a cohort study. The American journal of 454 medicine 2015;128(6):653.e7-.e16.

45516 White WB, Saag KG, Becker MA, et al. Cardiovascular Safety of Febuxostat or Allopurinol in 456 Patients with Gout. N Engl J Med 2018;378(13):1200-10. 

Hyperuricemic Heart Failure Patients: The Xanthine Oxidase Inhibition for Hyperuricemic Heart Failure Patients (EXACT-HF) Study. Circulation 2015;131(20):1763-71. not associate with beneficial cardiovascular outcomes: a population-based matched-cohort study. PLoS One 2014;9(6):e99102.

19 Gotsman I, Keren A, Lotan C, Zwas DR. Changes in uric acid levels and allopurinol use in chronic heart failure: association with improved survival. J Card Fail 2012;18(9):694-701.

20 MacDonald TM, Ford I, Nuki G, et al. Protocol of the Febuxostat versus Allopurinol Streamlined Trial (FAST): a large prospective, randomised, open, blinded endpoint study comparing the cardiovascular safety of allopurinol and febuxostat in the management of symptomatic hyperuricaemia. BMJ Open 2014;4(7):e005354.

21 Choi H, Neogi T, Stamp L, Dalbeth N, Terkeltaub R. New Perspectives in Rheumatology: Implications of the Cardiovascular Safety of Febuxostat and Allopurinol in Patients With Gout and Cardiovascular Morbidities Trial and the Associated Food and Drug Administration Public Safety Alert. Arthritis Rheumatol 2018;70(11):1702-9.

22 Singh JA, Cleveland J. Allopurinol and the risk of ventricular arrhythmias in the elderly: a study using US Medicare data. BMC Med 2017;15(1):59.

23 Johnson RJ, Bakris GL, Borghi C, et al. Hyperuricemia, Acute and Chronic Kidney Disease, Hypertension, and Cardiovascular Disease: Report of a Scientific Workshop Organized by the National Kidney Foundation. Am J Kidney Dis 2018;71(6):851-65. 24 Ho J, Dai RZW, Kwong TNY, et al. Disease Burden of Clostridium difficile Infections in Adults, Hong Kong, China, 2006-2014. Emerg Infect Dis 2017;23(10):1671-9. Microbes and Subsequent Diagnosis of Colorectal Cancer. Gastroenterology 2018;155(2):383-90 e8.

26 Chan EW, Lau WC, Leung WK, et al. Prevention of Dabigatran-Related Gastrointestinal Bleeding With Gastroprotective Agents: A Population-Based Study. Gastroenterology 2015;149(3):586-95 e3.

27 Man KK, Coghill D, Chan EW, et al. Methylphenidate and the risk of psychotic disorders and hallucinations in children and adolescents in a large health system. Transl Psychiatry 2016;6(11):e956.

28 Man KKC, Chan EW, Ip P, et al. Prenatal antidepressant use and risk of attentiondeficit/hyperactivity disorder in offspring: population based cohort study. BMJ 2017;357:j2350. 29 Wang SV, He M, Jin Y, et al. A review of the performance of different methods for propensity score matched subgroup analyses and a summary of their application in peer-reviewed research studies. Pharmacoepidemiol Drug Saf 2017;26(12):1507-12. 30 Wang SV, Jin Y, Fireman B, et al. Relative Performance of Propensity Score Matching Strategies for Subgroup Analyses. Am J Epidemiol 2018;187(8):1799-807. 31 Cuenca JA, Balda J, Palacio A, Young L, Pillinger MH, Tamariz L. Febuxostat and Cardiovascular Events: A Systematic Review and Meta-Analysis. Int J Rheumatol 2019;2019:1076189. 32 Zhang T, Pope JE. Cardiovascular effects of urate-lowering therapies in patients with chronic gout: a systematic review and meta-analysis. Rheumatology (Oxford) 2017;56(7):1144-53. 
501

502

503

504

505

506

507

508

509

510

511

512

513

514

515

516

517

518

519

520

521

522

523

524

525

526

527

528

529

530

531

532

533

534

535

536

inhibitors on cardiovascular disease in patients with gout: a cohort study. Am J Med 2015;128(6):653 e7- e16.

34 Richette P, Perez-Ruiz F, Doherty M, et al. Improving cardiovascular and renal outcomes in gout: what should we target? Nat Rev Rheumatol 2014;10(11):654-61.

35 Okafor ON, Farrington K, Gorog DA. Allopurinol as a therapeutic option in cardiovascular disease. Pharmacol Ther 2017;172:139-50.

36 Higgins P, Dawson J, Lees KR, McArthur K, Quinn TJ, Walters MR. Xanthine oxidase inhibition for the treatment of cardiovascular disease: a systematic review and meta-analysis. Cardiovasc Ther 2012;30(4):217-26.

37 White WB, Chohan S, Dabholkar A, Hunt B, Jackson R. Cardiovascular safety of febuxostat and allopurinol in patients with gout and cardiovascular comorbidities. Am Heart J 2012;164(1):14 20.

38 Choi H, Neogi T, Stamp L, Dalbeth N, Terkeltaub R. New Perspectives in Rheumatology: Implications of the Cardiovascular Safety of Febuxostat and Allopurinol in Patients With Gout and Cardiovascular Morbidities Trial and the Associated Food and Drug Administration Public Safety Alert. Arthritis \& Rheumatology 2018;70(11):1702-9.

39 Shin A, Kim MH, Ha Y-J, et al. OP0188 Effects of allopurinol versus febuxostat on cardiovascular risk in korean patients with gout: a nation-wide cohort study. Annals of the Rheumatic Diseases 2018;77(Suppl 2):143-.

40 Cicero AFG, Cosentino ER, Kuwabara M, Degli Esposti D, Borghi C. Effects of allopurinol and febuxostat on cardiovascular mortality in elderly heart failure patients. Intern Emerg Med 2019.

41 Malik UZ, Hundley NJ, Romero G, et al. Febuxostat inhibition of endothelial-bound XO: implications for targeting vascular ROS production. Free Radic Biol Med 2011;51(1):179-84.

42 Sezai A, Soma M, Nakata K, et al. Comparison of febuxostat and allopurinol for hyperuricemia in cardiac surgery patients with chronic kidney disease (NU-FLASH trial for CKD). J Cardiol 2015;66(4):298-303.

43 Edwards NL. Febuxostat: a new treatment for hyperuricaemia in gout. Rheumatology (Oxford) 2009;48 Suppl 2:ii15-ii9.

44 Deftereos S, Giannopoulos G, Panagopoulou V, et al. Anti-inflammatory treatment with colchicine in stable chronic heart failure: a prospective, randomized study. JACC Heart Fail 2014;2(2):131-7.

45 Hemkens LG, Ewald H, Gloy VL, et al. Colchicine for prevention of cardiovascular events. Cochrane Database Syst Rev 2016(1):CD011047.

46 Solomon DH, Liu CC, Kuo IH, Zak A, Kim SC. Effects of colchicine on risk of cardiovascular events and mortality among patients with gout: a cohort study using electronic medical records linked with Medicare claims. Ann Rheum Dis 2016;75(9):1674-9. 
539 Table 1. Baseline characteristics after propensity score matching.

540 Table 2. Crude incidence rates and hazard ratios for individual components of the

541 composite primary outcome and the secondary outcome comparing XOI users versus

542 XOI non-users after 1:1 matching.

543 Table 3. Crude incidence rates and hazard ratios for individual components of the 544 composite primary outcome and the secondary outcome comparing febuxostat users

545 versus allopurinol users after 1:3 matching.

546

547

548

549

550

551

552

553

554

\section{Figure Legends}

Figure 1. Selection process of the study cohort.

Figure 2. Kaplan-Meier curves comparing XOI users and non-users for (A) primary outcome and (B) all-cause mortality.

Figure 3. Kaplan-Meier curves comparing allopurinol and febuxostat users for (C) primary outcome and (D) all-cause mortality. 
Table 1. Baseline characteristics after 1:1 matching of XOI users to non-users, and 1:3 matching of febuxostat to allopurinol users.

\begin{tabular}{|c|c|c|c|c|c|c|c|c|}
\hline \multirow[b]{3}{*}{ Demogranhics } & \multicolumn{4}{|c|}{ After 1:1 matching } & \multicolumn{4}{|c|}{ After 1:3 matching } \\
\hline & $\begin{array}{c}\text { XOI users } \\
n=3607\end{array}$ & $\begin{array}{l}\text { XOI non- } \\
\text { users } \\
n=3607\end{array}$ & P-value & SMD & $\begin{array}{c}\text { Febuxostat } \\
\text { users } \\
n=276\end{array}$ & $\begin{array}{c}\text { Allopurinol } \\
\text { users } \\
n=828\end{array}$ & P-value & SMD \\
\hline & & & & & & & & \\
\hline Male & $2563(71.1)$ & $2546(70.6)$ & 0.679 & 0.010 & $186(67.4)$ & $549(66.3)$ & 0.797 & 0.023 \\
\hline Age & $71.46(14.59)$ & $72.12(15.36)$ & 0.061 & 0.044 & $70.41(14.35)$ & $70.01(14.90)$ & 0.703 & 0.027 \\
\hline \multicolumn{9}{|l|}{ Comorbidity } \\
\hline PVD & $50(1.4)$ & $56(1.6)$ & 0.625 & 0.014 & $4(1.4)$ & $16(1.9)$ & 0.794 & 0.037 \\
\hline Rheumatic disease & $30(0.8)$ & $35(1.0)$ & 0.618 & 0.015 & $5(1.8)$ & $11(1.3)$ & 0.771 & 0.039 \\
\hline Liver disease & $24(0.7)$ & $19(0.5)$ & 0.541 & 0.018 & $3(1.1)$ & $12(1.4)$ & 0.881 & 0.032 \\
\hline $\mathrm{DM}$ & $681(18.9)$ & $663(18.4)$ & 0.607 & 0.013 & $59(21.4)$ & $185(22.3)$ & 0.802 & 0.023 \\
\hline Renal disease & $527(14.6)$ & $519(14.4)$ & 0.815 & 0.006 & $46(16.7)$ & $139(16.8)$ & 1.000 & 0.003 \\
\hline Charlson's score & $\begin{array}{c}0.00 \\
{[0.00,1.00]}\end{array}$ & $\begin{array}{c}0.00 \\
{[0.00,1.00]}\end{array}$ & 0.535 & 0.013 & $\begin{array}{c}0.00 \\
{[0.00,2.00]}\end{array}$ & $\begin{array}{c}0.00 \\
{[0.00 .2 .00]}\end{array}$ & 0.632 & 0.030 \\
\hline \multicolumn{9}{|l|}{$\begin{array}{l}\text { Cardiovascular } \\
\text { medications }\end{array}$} \\
\hline Lipid-lowering drugs & $1501(41.6)$ & $1505(41.7)$ & 0.943 & 0.002 & $123(44.6)$ & $374(45.2)$ & 0.917 & 0.012 \\
\hline ACE inhibitors/ARBs & $1514(42.0)$ & $1521(41.4)$ & 0.633 & 0.012 & $131(47.5)$ & $402(48.6)$ & 0.808 & 0.022 \\
\hline Antidiabetic drugs & $798(22.1)$ & $779(21.6)$ & 0.608 & 0.013 & $82(29.7)$ & $256(30.9)$ & 0.763 & 0.026 \\
\hline Diuretics & $1099(30.5)$ & $1074(29.8)$ & 0.538 & 0.015 & $108(39.1)$ & $328(39.6)$ & 0.943 & 0.010 \\
\hline Beta-blockers & $1412(39.1)$ & $1482(41.1)$ & 0.097 & 0.040 & $117(42.4)$ & $373(45.0)$ & 0.484 & 0.054 \\
\hline Nitrates & $320(8.9)$ & $314(8.7)$ & 0.835 & 0.006 & $17(6.2)$ & $50(6.0)$ & 1.000 & 0.005 \\
\hline $\mathrm{CCBs}$ & $2081(57.7)$ & $2103(58.3)$ & 0.616 & 0.012 & $160(58.0)$ & $471(56.9)$ & 0.806 & 0.022 \\
\hline Anticoagulants/ & $1368(37.9)$ & $1382(38.3)$ & 0.753 & 0.008 & $99(35.9)$ & $285(34.4)$ & 0.715 & 0.030 \\
\hline Antiplatelet agents & & & & & & & & \\
\hline \multicolumn{9}{|l|}{ Other gout medications } \\
\hline Colchicine & 2628 (72.9) & $2649(73.4)$ & 0.595 & 0.013 & 237 (85.9) & 716 (86.5) & 0.879 & 0.017 \\
\hline Probenecid & $9(0.2)$ & $9(0.2)$ & 1.000 & $<0.001$ & $7(2.5)$ & $2(0.2)$ & 0.001 & 0.197 \\
\hline NSAIDs & $2063(57.2)$ & $2036(56.4)$ & 0.537 & 0.015 & $159(57.6)$ & $485(58.6)$ & 0.833 & 0.020 \\
\hline
\end{tabular}

All data was presented in number (percentage) or median [interquartile range] as appropriate. XOI, xanthine oxidase inhibitors; ACE, angiotensin converting enzyme; ARB, angiotensin II receptor blockers; CCB, calcium channel blockers; DM, diabetes mellitus; IQR, interquartile range; PVD, peripheral vascular disease; NSAID, non-steroid anti-inflammatory drugs. 
Table 2. Adjusted hazard ratios for the composite primary and secondary outcomes comparing XOI users to non-users.

\begin{tabular}{|c|c|c|c|c|c|c|}
\hline Outcome & $\begin{array}{l}\text { XOI users } \\
(n=3607) \\
\text { Event }(n)\end{array}$ & $\begin{array}{c}\text { XOI users } \\
\text { Crude IR (per } \\
1000 \text { patient- } \\
\text { years) }\end{array}$ & $\begin{array}{l}\text { XOI non-users } \\
(\mathrm{n}=3067) \\
\text { Event }(\mathrm{n})\end{array}$ & $\begin{array}{l}\text { XOI non-users } \\
\text { Crude IR (per } \\
1000 \text { patient- } \\
\text { years) }\end{array}$ & $\operatorname{HR}(95 \% \mathrm{CI})$ & P-value \\
\hline \multicolumn{7}{|l|}{ Primary outcome } \\
\hline $\begin{array}{l}\text { Composite out- } \\
\text { come }\end{array}$ & 497 & 62.77 & 487 & 66.13 & $0.997(0.879-1.131)$ & 0.962 \\
\hline Nonfatal MI & 115 & 14.50 & 99 & 13.15 & $1.111(0.849-1.453)$ & 0.444 \\
\hline Stroke & 117 & 14.75 & 139 & 18.64 & $0.821(0.640-1.054)$ & 0.121 \\
\hline $\begin{array}{l}\text { Cardiovascular } \\
\text { death }\end{array}$ & 5 & 0.63 & 7 & 0.93 & $0.680(0.216-2.145)$ & 0.511 \\
\hline $\begin{array}{l}\text { Hospitalization due } \\
\text { to } \mathrm{CHF}\end{array}$ & 287 & 36.18 & 274 & 36.39 & $1.028(0.870-1.215)$ & 0.743 \\
\hline \multicolumn{7}{|l|}{$\begin{array}{l}\text { Secondary out- } \\
\text { come }\end{array}$} \\
\hline All-cause mortality & 922 & 106.90 & 913 & 111.35 & $0.972(0.886-1.065)$ & 0.539 \\
\hline
\end{tabular}


Table 3. Adjusted hazard ratios for the primary and secondary outcomes comparing febuxostat users to allopurinol users.

\begin{tabular}{|c|c|c|c|c|c|c|}
\hline Outcome & $\begin{array}{c}\text { Febuxostat us- } \\
\text { ers } \\
(\mathrm{n}=276) \\
\text { Event }(\mathrm{n})\end{array}$ & $\begin{array}{l}\text { Febuxostat users } \\
\text { Crude IR (per } \\
1000 \text { patient- } \\
\text { years) }\end{array}$ & $\begin{array}{l}\text { Allopurinol users } \\
(\mathrm{n}=828) \\
\text { Event }(\mathrm{n})\end{array}$ & $\begin{array}{l}\text { Allopurinol users } \\
\text { Crude IR (per } \\
1000 \text { patient- } \\
\text { years) }\end{array}$ & $\operatorname{HR}(95 \% \mathrm{CI})$ & P-value \\
\hline \multicolumn{7}{|l|}{$\begin{array}{l}\text { Primary out- } \\
\text { come }\end{array}$} \\
\hline $\begin{array}{l}\text { Composite out- } \\
\text { come }\end{array}$ & 21 & 43.41 & 107 & 59.53 & $0.672(0.416-1.085)$ & 0.104 \\
\hline Nonfatal MI & 4 & 8.27 & 19 & 10.57 & $0.789(0.267-2.329)$ & 0.667 \\
\hline Stroke & 6 & 12.40 & 25 & 13.91 & $0.891(0.363-2.185)$ & 0.800 \\
\hline $\begin{array}{l}\text { Cardiovascular } \\
\text { death }\end{array}$ & 0 & 0 & 0 & 0 & - & - \\
\hline $\begin{array}{l}\text { Hospitalization } \\
\text { due to CHF } \\
\text { Secondary out- } \\
\text { come }\end{array}$ & 11 & 22.74 & 67 & 37.28 & $0.529(0.272-1.029)$ & 0.061 \\
\hline $\begin{array}{l}\text { All-cause mortal- } \\
\text { ity }\end{array}$ & 52 & 104.27 & 204 & 105.49 & $0.985(0.725-1.338)$ & 0.921 \\
\hline
\end{tabular}

